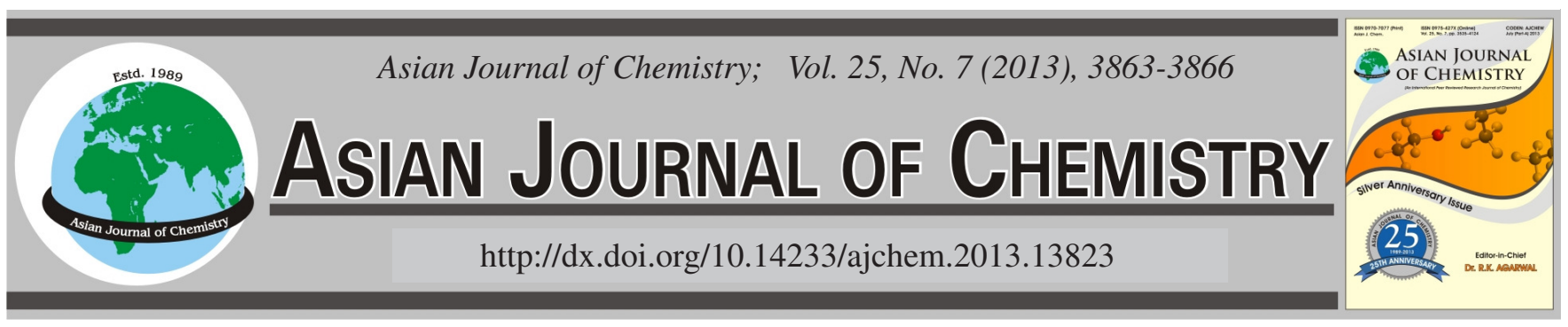

\title{
Study on the Structure-Activity Relations of Brominated Hydroxy Diphenyl Ethers Derivatives with Anilines
}

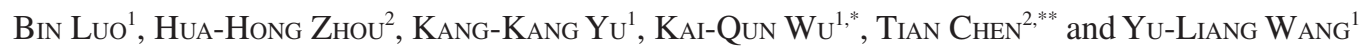

${ }^{1}$ Faculty of Chemistry, Sichuan University, Chengdu 610065, P.R. China

${ }^{2}$ Department of Pathogenic Biology, School of Biomedical Science, Chengdu Medical College, Chengdu 610083, P.R. China

"Corresponding author: Tel: +86 13708058490, E-mail: wukaiqun@tom.com; luobinhxscu@126.com

${ }^{* *}$ Corresponding author (For biological activities assay): Tel: +86 13540047681, E-mail: tianchen66@ gmail.com

(Received: 3 April 2012;

Accepted: 14 January 2013)

AJC-12712

\begin{abstract}
In order to study the influence of anilines on antibacterial activity, eight novel brominated hydroxy diphenyl ethers derivatives were designed and synthesized. The antibacterial activities of the new compounds were tested via agar-well diffusion method in vitro under different concentrations. The results showed the derivatives had antibacterial activities at the concentration $50 \mu \mathrm{g} / \mathrm{mL}$ against Staphylococcus aureus SC and Staphylococcus aureus ATCC26112.

Key Words: Brominated hydroxy diphenyl ethers, Structure-activity, Anilines, Antibacterial activity.
\end{abstract}

ᄂ - - - - - - - - - - - - - - - -

\section{INTRODUCTION}

Brominated 2-hydroxy diphenyl ethers was first isolated from the marine sponge Dysidea herbacea by Sharma et al. ${ }^{1}$ (Fig. 1, 1) and it was reported that brominated 2-hydroxy diphenyl ethers could inhibit gram positive bacteria and gram negative bacteria ${ }^{1,2}$. Afterwards, many literatures have focued on the isolation and synthesise new brominated hydroxy diphenyl ethers and their antibacterial activities ${ }^{3-5}$. These studies showed that the hydroxy was necessary for keeping antibacterial activity $^{6,7}$ and fluorine as well as nitro substituent were favourable for enhancing antibacterial activity ${ }^{8,9}$. They also emplyed that some synthetic derivatives had a special antibacterial activities such as a series of brominated dihydroxy diphenyl ethers derivatives have been designed and synthesized with good antibacterial activities ${ }^{10,11}$ (Fig. 1, 2). However, dioxins were found during the separation of natural brominated 2hydroxy diphenyl ethers ${ }^{12,13}$ (Fig. 1, 3). In order to avoid the generation of dioxins a series of brominated 3-hydroxy (Fig. 1, 4) and 4-hydroxy (Fig. 1, 5) diphenyl ethers derivatives were designed and synthesized. The results showed that brominated 3-hydroxy and 4-hydroxy diphenyl ethers derivatives had also a good antibacterial activities and will never form dioxins $^{14,15}$.

In order to avoid the generation of dioxins and to study the influence of anilines on antibacterial activity systematically, a series of novel brominated 4-hydroxy diphenyl ethers derivatives containing aniline have been designed and synthesized. The antibacterial activities of the target compounds have been tested via the agar-well diffusion method in vitro. The results showed that target compounds had some antibacterial activities against Staphylococcus aureus SC, Staphylococcus aureus ATCC26112. The synthetic route is shown in Scheme-I.<smiles>[R]c1ccc(Oc2c([R9])c([R6])c([R6])c(O)c2[R])c([R])c1</smiles>

$R_{3}=H, R_{4}=R_{5}=R_{6}=R_{2}^{\prime}=R_{4}^{\prime}=B r$ $R_{6}=H, R_{3}=R_{4}=R_{5}=R_{2}^{\prime}=R_{4}^{\prime}=B r$ $R_{4}=R_{6}=H, R_{3}=R_{5}=R_{2}=R_{4}^{\prime}=B r$ $R_{3}=R_{5}=R_{2}^{\prime}=H, R_{4}=R_{6}=R_{4}^{\prime}=B r$ $R_{3}=R_{4}=R_{5}=R_{2}^{\prime}=H, R_{6}=R_{4}^{\prime}=B r$<smiles>[X]c1[R]cc(Oc2ccc(Br)c(O)c2)cc1</smiles>

The formation of dioxins

3

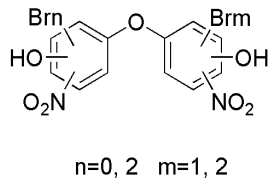

2

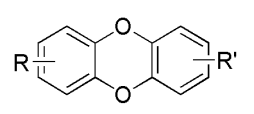<smiles>[R]c1cc(OC2=CC=C(O)[Se]C=C2)cc[n+]1[O-]</smiles>

5 4

Fig. 1. Brominated hydroxy diphenyl ethers and related derivatives 


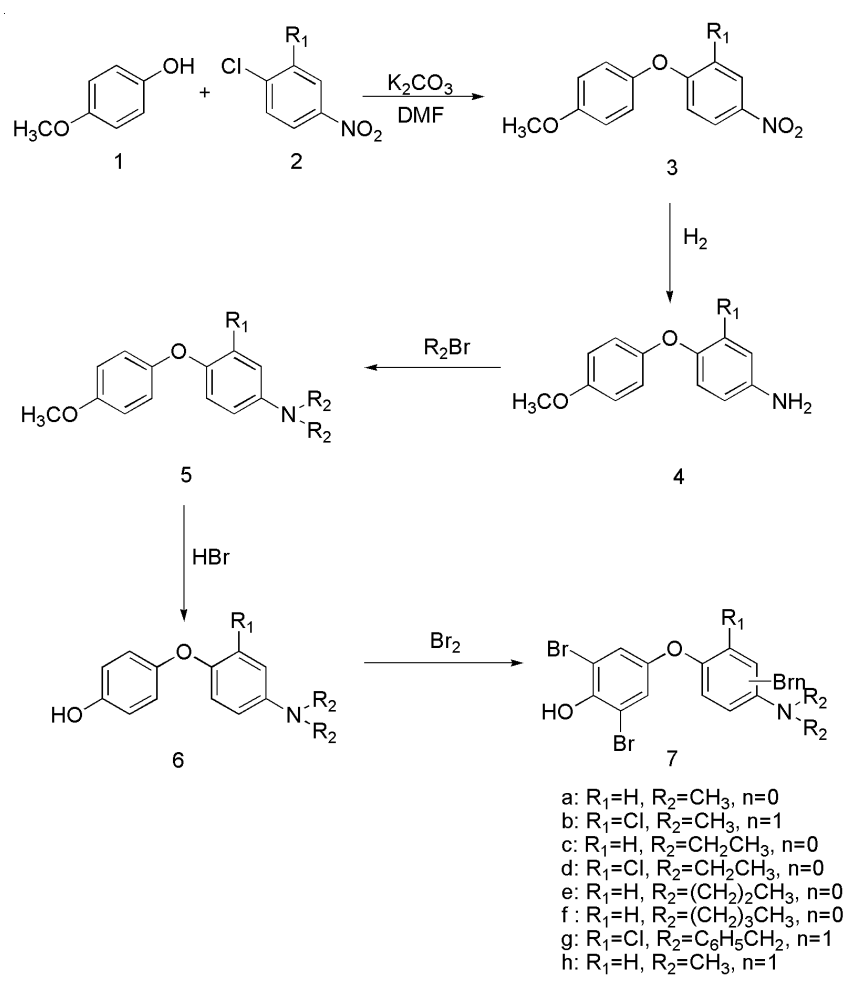

Scheme-I: Synthetic route of compounds $\mathbf{7 a - 7 h}$

\section{EXPERIMENTAL}

Melting points were recorded on an XRC-1 melting point apparatus (Sichuan University Instrument Inc., Chengdu, China) without being corrected. ${ }^{1} \mathrm{H} \mathrm{NMR}$ spectra were run on a Varian INOVA-400 spectrometer (Varian Inc., Palo Alto, CA, USA) with $\mathrm{CDCl}_{3}$ as the solvent and TMS as the internal standard. Mass spectra were recorded with an Agilent 6210 (DOF-MAS) spectrometer (Agilent Inc., Santa Clara, CA, USA) using the electrospray ionization (ESI) method. IR spectra were recorded with a Perkin-Elmer 16PC-FT instrument (Perkin-Elmer Inc., Norwalk Conn, CA, USA). Compounds 1 and $\mathbf{2}$ were commercially available, compounds $\mathbf{3}$ and $\mathbf{4}$ were synthesized according to the literature ${ }^{16}$.

General preparation procedure of 5a-b: A mixture of 4 (1 mmol) and $\mathrm{NaOH}(5 \mathrm{mmol})$ in solvent $(20 \mathrm{~mL}$, dry ethanol: water $=3: 1$ ) was stirred at $0-5^{\circ} \mathrm{C}$, then dimethyl sulfate $(6 \mathrm{mmol})$ was added dropwise. Reaction solution was stirred at $0-15^{\circ} \mathrm{C}$ for $24 \mathrm{~h}$. Then the $\mathrm{pH}$ was adjusted to 7 with diluted hydrochloric acid, $20 \mathrm{~mL}$ water was added and the mixture was extracted with ethyl acetate. The organic layer was filtered, dried and evaporated in vacuo to give the crude product. The crude product was chromatographed on silica gel using petroleum ether/ethyl acetate (16:1) as eluent to afford a pure product (yield 50-55\%).

General preparation procedure of 5c-g: A mixture of 4 (1 mmol), dry $\mathrm{Na}_{2} \mathrm{CO}_{3}(3 \mathrm{mmol})$ and $\mathrm{RBr}(3-6 \mathrm{mmol})$ in dry DMF (20 mL) was stirred at $60 \sim 110^{\circ} \mathrm{C}$ for $3 \sim 24 \mathrm{~h}$, cooled. $20 \mathrm{~mL}$ water was added and the mixture was extracted with ethyl acetate. The organic layer was filtered, dried and evaporated in vacuo to give the crude product. The crude product was chromatographed on silica gel using petroleum ether/ethyl acetate $(16: 1)$ as eluent to afford a pure product (yield 60$92 \%)$.
General preparation procedure of $6 \mathrm{a}-\mathrm{g}$ : A mixture of 5a-g $(1 \mathrm{mmol})$ and $40 \% \mathrm{HBr}(6 \mathrm{mmol})$ in acetic acid $(20 \mathrm{~mL})$ was refluxed at $125^{\circ} \mathrm{C}$ for $9 \sim 11 \mathrm{~h}$, cooled, the $\mathrm{pH}$ was adjusted to 7 with saturated $\mathrm{NaHCO}_{3}$ solution and the mixture was extracted with ethyl acetate. The organic layer was filtered, dried and evaporated in vacuo to give the crude product. The crude product was chromatographed on silica gel using petroleum ether/ethyl acetate $(4: 1)$ as eluent to afford a pure product (yield 45-85\%).

General preparation procedure of 7a-h: 6a-g (1 mmol) was dissolved in ethyl acetate/dichloromethane $(1: 1,20 \mathrm{~mL})$ and the mixture was heated to reflux. Liquid bromide (6 mmol ) was dissolved in ethyl acetate/dichloromethane (1:1, $4 \mathrm{~mL})$ and the solution was added dropwise. The reaction mixture was refluxed for $7 \mathrm{~h}$, cooled, the reaction mixture was washed by $5 \% \mathrm{Na}_{2} \mathrm{~S}_{2} \mathrm{O}_{3}$, saturated $\mathrm{NaHCO}_{3}$ solution, water. The organic layer was filtered, dried and evaporated in vacuo to give the crude product. The crude product was chromatographed on silica gel using petroleum ether/ethyl acetate $(20: 1)$ as eluent to afford a pure product (yield 45-55 \%).

5a, 5c are known compounds reported in literature ${ }^{17}$ and were identified by melting point test, ${ }^{1} \mathrm{H}$ NMR and IR, all data was the same with the literature.

3-Chloro-4-(4-methoxyphenoxy)- $\mathbf{N}, \boldsymbol{N}$-dimethylbenzen-amine (5b): Yellow solid; yield:52.3\%, m.p.: 52-54 ${ }^{\circ} \mathrm{C}$; ${ }^{1} \mathrm{H}$ NMR spectrum (400 MHz; $\left.\mathrm{d}_{1}-\mathrm{CDCl}_{3} ; \mathrm{TMS}\right): \delta$ (ppm) $=2.93(6 \mathrm{H}, \mathrm{s}), 3.77(3 \mathrm{H}, \mathrm{s}), 6.58\left(1 \mathrm{H}, \mathrm{dd}, J_{1}=8.8 \mathrm{~Hz}, J_{2}=2.8\right.$ $\mathrm{Hz}), 6.77(1 \mathrm{H}, \mathrm{d}, J=2.8 \mathrm{~Hz}), 6.81-7.03(5 \mathrm{H}, \mathrm{m})$; IR $(\mathrm{KBr}$, $\left.V_{\max }, \mathrm{cm}^{-1}\right) 3040,2936,2834,1607,1562,1499,1443,1234$, 1202, 1039, 961, 879, 828, 772.

3-Chloro-4-(4-methoxyphenoxy)- $N$, $N$-diethylbenzenamine (5d): Yellow oilyliquid; yield: $80.2 \%$; ${ }^{1} \mathrm{H} \mathrm{NMR}$ spectrum (400 MHz; $\left.\mathrm{d}_{1}-\mathrm{CDCl}_{3} ; \mathrm{TMS}\right): \delta(\mathrm{ppm})=0.93(6 \mathrm{H}, \mathrm{t}$, $J=7.6 \mathrm{~Hz}), 3.19(4 \mathrm{H}, \mathrm{q}, J=7.6 \mathrm{~Hz}), 3.77(3 \mathrm{H}, \mathrm{s}), 6.53(1 \mathrm{H}, \mathrm{dd}$, $\left.J_{1}=8.8 \mathrm{~Hz}, J_{2}=2.8 \mathrm{~Hz}\right), 6.66(1 \mathrm{H}, \mathrm{d}, J=2.8 \mathrm{~Hz}), 6.81-7.00$ $(5 \mathrm{H}, \mathrm{m})$; IR $\left(\mathrm{KBr}, v_{\max }, \mathrm{cm}^{-1}\right) 3046,2970,2835,1607,1556$, 1498, 1376, 1356, 1232, 1200, 1039, 874, 826, 794, 756.

4-(4-Methoxyphenoxy)- $\boldsymbol{N}, \boldsymbol{N}$-dipropylbenzenamine (5e): Yellow oilyliquid; yield: 65.3\%; ${ }^{1} \mathrm{H}$ NMR spectrum (400 $\mathrm{MHz} ; \mathrm{d}_{1}-\mathrm{CDCl}_{3}$; TMS $): \delta(\mathrm{ppm})=0.92(6 \mathrm{H}, \mathrm{t}, J=7.6 \mathrm{~Hz})$, $1.59(4 \mathrm{H}, \mathrm{m}), 3.19(4 \mathrm{H}, \mathrm{t}, J=7.6 \mathrm{~Hz}), 3.83(3 \mathrm{H}, \mathrm{s}), 6.60(2 \mathrm{H}, \mathrm{d}$, $J=8.8 \mathrm{~Hz}) ; 6.81-6.92(6 \mathrm{H}, \mathrm{m})$; IR $\left(\mathrm{KBr}, \mathrm{v}_{\max }, \mathrm{cm}^{-1}\right): 3045$, 2960, 2873, 2835, 1612, 1500, 1462, 1370, 1225, 1100, 1037, $873,824,746$.

4-(4-Methoxyphenoxy)- $\boldsymbol{N}, \boldsymbol{N}$-dibutyl-benzenamine (5f): Yellow oily liquid; yield: $60.3 \%$; ${ }^{1} \mathrm{H}$ NMR spectrum (400 $\mathrm{MHz} ; \mathrm{d}_{1}-\mathrm{CDCl}_{3}$; TMS): $\delta(\mathrm{ppm})=0.95(6 \mathrm{H}, \mathrm{t}, J=7.2 \mathrm{~Hz})$, $1.34(4 \mathrm{H}, \mathrm{m}), 1.50(4 \mathrm{H}, \mathrm{m}), 3.22(4 \mathrm{H}, \mathrm{t}, J=7.6 \mathrm{~Hz}), 3.80(3 \mathrm{H}$, s), $6.60(2 \mathrm{H}, \mathrm{d}, J=8.8 \mathrm{~Hz}), 6.81-6.90(6 \mathrm{H}, \mathrm{m})$; IR (KBr, $\mathrm{v}_{\max }$, $\mathrm{cm}^{-1}$ ): 3044, 2957, 2868, 1611, 1500, 1462, 1369, 1277, 1227, $1105,1037,824$.

3-Chloro-4-(4-methoxyphenoxy)- $N, N$-dibenzylbenzenamine (5g): Colourless oilyliquid; yield: $91.3 \%$; ${ }^{1} \mathrm{H}$ NMR spectrum (400 MHz; $\left.\mathrm{d}_{1}-\mathrm{CDCl}_{3} ; \mathrm{TMS}\right): \delta(\mathrm{ppm})=3.77$ $(3 \mathrm{H}, \mathrm{s}), 4.67(4 \mathrm{H}, \mathrm{s}), 6.56\left(1 \mathrm{H}, \mathrm{dd}, J_{1}=8.8 \mathrm{~Hz}, J_{2}=3.2 \mathrm{~Hz}\right)$, 6.79-6.91 (6H, m), 7.07-7.39 (10H, m); IR (KBr, $\left.v_{\max }, \mathrm{cm}^{-1}\right)$ : 3029, 2946, 2833, 1604, 1497, 1452, 1393, 1361, 1230, 1200, 1030, 957, 883, 821, 733, 696. 
4-[4-(Dimethylamino)phenoxy]phenol (6a): White solid; yield: $84.7 \%$, m.p.: $133-135{ }^{\circ} \mathrm{C} ;{ }^{1} \mathrm{H}$ NMR spectrum $\left(400 \mathrm{MHz} ; \mathrm{d}_{1}-\mathrm{CDCl}_{3} ; \mathrm{TMS}\right): \delta(\mathrm{ppm})=2.93(6 \mathrm{H}, \mathrm{s}), 4.61$ $(1 \mathrm{H}, \mathrm{s}), 6.76(2 \mathrm{H}, \mathrm{d}, J=8.4 \mathrm{~Hz}), 6.85(4 \mathrm{H}, \mathrm{d}, J=8.8 \mathrm{~Hz}), 6.92$ $(2 \mathrm{H}, \mathrm{d}, J=8.8 \mathrm{~Hz})$; IR $\left(\mathrm{KBr}, v_{\max }, \mathrm{cm}^{-1}\right): 3430,3043,2895$, 2813, 1603, 1502, 1451, 1366, 1303, 1227, 1145, 1096, 1051, $932,871,826$.

4-[2-Chloro-4-(dimethylamino)phenoxy]phenol (6b): White solid; yield: $83.6 \%$, m.p.: $127-129{ }^{\circ} \mathrm{C}$; ${ }^{1} \mathrm{H}$ NMR spectrum $\left(400 \mathrm{MHz} ; \mathrm{d}_{1}-\mathrm{CDCl}_{3} ; \mathrm{TMS}\right): \delta(\mathrm{ppm})=2.94(6 \mathrm{H}, \mathrm{s})$, $4.53(1 \mathrm{H}, \mathrm{s}), 6.61-6.83(5 \mathrm{H}, \mathrm{m}), 6.91(2 \mathrm{H}, \mathrm{d}, J=8.8 \mathrm{~Hz})$; IR $\left(\mathrm{KBr}, v_{\max }, \mathrm{cm}^{-1}\right)$ 3097, 2921, 2821, 1600, 1495, 1443, 1355, $1237,1142,1052,948,879,822,782,509$.

4-[4-(Diethylamino)phenoxy]phenol (6c): White solid; yield: $82.0 \%$, m.p.: $113-115^{\circ} \mathrm{C} ;{ }^{1} \mathrm{H}$ NMR spectrum $(400 \mathrm{MHz}$; $\mathrm{d}_{1}-\mathrm{CDCl}_{3}$; TMS $): \delta(\mathrm{ppm})=1.16(6 \mathrm{H}, \mathrm{t}, J=3.6 \mathrm{~Hz}), 3.31(4 \mathrm{H}$, q, $J=6.8 \mathrm{~Hz}), 4.87(1 \mathrm{H}, \mathrm{s}), 6.76(2 \mathrm{H}, \mathrm{d}, J=8.8 \mathrm{~Hz}), 6.86(4 \mathrm{H}$, $\mathrm{d}, J=8.8 \mathrm{~Hz}), 6.89(2 \mathrm{H}, \mathrm{d}, J=9.2 \mathrm{~Hz})$; IR $\left(\mathrm{KBr}, v_{\max }, \mathrm{cm}^{-1}\right)$ : 3385, 3041, 2972, 2931, 1607, 1500, 1448, 1354, 1224, 1094, 1010, 872, 820 .

4-[2-Chloro-4-(diethylamino)phenoxy]phenol (6d): Yellow oilyliquid; yield: $79.5 \% ;{ }^{1} \mathrm{H}$ NMR spectrum $(400 \mathrm{MHz}$; $\mathrm{d}_{1}-\mathrm{CDCl}_{3}$; TMS $): \delta(\mathrm{ppm})=1.16(6 \mathrm{H}, \mathrm{t}, J=7.2 \mathrm{~Hz}), 3.32(4 \mathrm{H}$, q, $J=7.2 \mathrm{~Hz}), 4.58(1 \mathrm{H}, \mathrm{s}), 6.53\left(1 \mathrm{H}, \mathrm{dd}, J_{1}=8.8 \mathrm{~Hz}, J_{2}=2.8\right.$ $\mathrm{Hz}), 6.70(1 \mathrm{H}, \mathrm{d}, J=2.8 \mathrm{~Hz}), 6.75(1 \mathrm{H}, \mathrm{d}, J=9.2 \mathrm{~Hz}), 6.80$ $(2 \mathrm{H}, \mathrm{d}, J=8.8 \mathrm{~Hz}), 6.89(2 \mathrm{H}, \mathrm{d}, J=8.8 \mathrm{~Hz})$; IR $\left(\mathrm{KBr}, \mathrm{v}_{\max }\right.$, $\mathrm{cm}^{-1}$ ): 3390, 3033, 2972, 2931, 1605, 1557, 1505, 1356, 1233, 1096, 1045, 1013, 924, 874, 828, 768, 693, 607, 512.

4-[4-(Dipropylamino)phenoxy]phenol (6e): Yellow oilyliquid; yield: $73.8 \%$; ${ }^{1} \mathrm{H}$ NMR spectrum $\left(400 \mathrm{MHz} ; \mathrm{d}_{1-}\right.$ $\mathrm{CDCl}_{3}$; TMS $): \delta(\mathrm{ppm})=0.92(6 \mathrm{H}, \mathrm{t}, J=7.6 \mathrm{~Hz}), 1.59(4 \mathrm{H}$, m), $3.19(4 \mathrm{H}, \mathrm{t}, J=7.6 \mathrm{~Hz}), 4.60(1 \mathrm{H}, \mathrm{s}), 6.60(2 \mathrm{H}, \mathrm{d}, J=8.8$ $\mathrm{Hz}) ; 6.75(2 \mathrm{H}, \mathrm{d}, J=8.8 \mathrm{~Hz}), 6.84-6.87(4 \mathrm{H}, \mathrm{m})$; IR $(\mathrm{KBr}$, $\left.V_{\max }, \mathrm{cm}^{-1}\right): 3404,3046,2962,2931,2873,1608,1500,1460$, 1364, 1222, 1098, 1046, 873, 818,757.

4-[4-(Dibutylamino)phenoxy]phenol (6f): Yellow oilyliquid; yield: $70.1 \%$; ${ }^{1} \mathrm{H}$ NMR spectrum $\left(400 \mathrm{MHz} ; \mathrm{d}_{1}-\right.$ $\mathrm{CDCl}_{3}$; TMS): $\delta(\mathrm{ppm})=0.95(6 \mathrm{H}, \mathrm{t}, J=7.2 \mathrm{~Hz}), 1.34(4 \mathrm{H}$, m), $1.50(4 \mathrm{H}, \mathrm{m}), 3.22(4 \mathrm{H}, \mathrm{t}, J=7.6 \mathrm{~Hz}), 6.61(2 \mathrm{H}, \mathrm{d}, J=8.4$ $\mathrm{Hz}), 6.75(2 \mathrm{H}, \mathrm{d}, J=8.8 \mathrm{~Hz}), 6.85-6.89(4 \mathrm{H}, \mathrm{m})$; IR $(\mathrm{KBr}$, $\left.V_{\max }, \mathrm{cm}^{-1}\right): 3044,2957,2868,1611,1500,1462,1369,1277$, $1227,1105,1037,824$.

4-[2-Chloro-4-(dibenzylamino)phenoxy]phenol (6g): Colourless oilyliquid; yield: $45.3 \%$; ${ }^{1} \mathrm{H}$ NMR spectrum (400 $\mathrm{MHz} ; \mathrm{d}_{1}-\mathrm{CDCl}_{3}$; TMS): $\delta(\mathrm{ppm})=4.30(4 \mathrm{H}, \mathrm{s}), 4.60(1 \mathrm{H}, \mathrm{s})$, $6.63(1 \mathrm{H}, \mathrm{d}, J=8.8 \mathrm{~Hz}), 6.76(1 \mathrm{H}, \mathrm{d}, J=3.2 \mathrm{~Hz}), 6.88-7.01$ $(5 \mathrm{H}, \mathrm{m}), 7.28-7.39(10 \mathrm{H}, \mathrm{m})$; IR $\left(\mathrm{KBr}, \mathrm{v}_{\max }, \mathrm{cm}^{-1}\right)$ : 3335, 3030, 2874, 1604, 1504, 1449, 1357, 1250, 1095, 1053, 1006, 959, 884, 836, 801, 737, 698, 405.

2,6-Dibromo-4-[4-(dimethylamino)phenoxy]phenol (7a): Yellow solid; yield:15.3\%; m.p.: $126-128{ }^{\circ} \mathrm{C}$; ${ }^{1} \mathrm{H}$ NMR spectrum (400 MHz; $\left.\mathrm{d}_{1}-\mathrm{CDCl}_{3} ; \mathrm{TMS}\right): \delta(\mathrm{ppm})=2.78(6 \mathrm{H}$, s), $5.36(1 \mathrm{H}, \mathrm{s}), 6.88(2 \mathrm{H}, \mathrm{d}, J=2.8 \mathrm{~Hz}), 7.01(2 \mathrm{H}, \mathrm{d}, J=5.6$ $\mathrm{Hz})$, 7.16 (2H, s); IR (KBr, $\left.v_{\max }, \mathrm{cm}^{-1}\right) 3503,3069,2929,2866$, 2784, 1592, 1498, 1415, 1324, 1267, 1212, 1185, 1034, 916, 883, 817, 781, 569; HR-MS (ESI): Calcd. for $\mathrm{C}_{14} \mathrm{H}_{14} \mathrm{NO}_{2} \mathrm{Br}_{2}$ $[\mathrm{M}+\mathrm{H}]^{+}$: 385.9392, 387.9372, 389.9351; Found: 385.9381, $387.9363,389.9345$.
2,6-Dibromo-4-[3-bromo-5-chloro-4-(dimethylamino)phenoxy]phenol (7b): White solid; yield: $47.7 \%$, m.p.: 109$111{ }^{\circ} \mathrm{C} ;{ }^{1} \mathrm{H}$ NMR spectrum (400 MHz; $\mathrm{d}_{1}-\mathrm{CDCl}_{3}$; TMS): $\delta$ $(\mathrm{ppm})=2.80(6 \mathrm{H}, \mathrm{s}), 5.68(1 \mathrm{H}, \mathrm{s}), 7.08(1 \mathrm{H}, \mathrm{s}), 7.15(1 \mathrm{H}, \mathrm{s})$, $7.20(1 \mathrm{H}, \mathrm{s}), 7.30(1 \mathrm{H}, \mathrm{s})$; IR (KBr, $\left.v_{\max }, \mathrm{cm}^{-1}\right)$ : 3284, 3081, 2960, 2873, 2841, 2797, 1593, 1564, 1464, 1400, 1355, 1317, 1216, 1184, 1134, 1072, 1045, 970, 932, 887, 852, 801, 768, 733, 681, 580, 517, 466, 422; HR-MS (ESI): Calcd. for $\mathrm{C}_{14} \mathrm{H}_{12} \mathrm{NO}_{2} \mathrm{Br}{ }_{3} \mathrm{Cl}[\mathrm{M}+\mathrm{H}]^{+}:$497.8107, 499.8087, 501.8067; Found: 497.8091,499.8069, 501.8048.

2,6-Dibromo-4-[4-(diethylamino)phenoxy]phenol (7c): White solid; yield: $48.9 \%$, m.p.: $103-105{ }^{\circ} \mathrm{C} ;{ }^{1} \mathrm{H}$ NMR spectrum (400 MHz; $\left.\mathrm{d}_{1}-\mathrm{CDCl}_{3} ; \mathrm{TMS}\right): \delta(\mathrm{ppm})=1.17(6 \mathrm{H}, \mathrm{t}, J=$ $6.8 \mathrm{~Hz}), 3.34(4 \mathrm{H}, \mathrm{q}, J=6.8 \mathrm{~Hz}), 5.58(1 \mathrm{H}, \mathrm{s}), 6.66(2 \mathrm{H}, \mathrm{d}, J$ $=8.8 \mathrm{~Hz}), 6.88(2 \mathrm{H}, \mathrm{d}, J=9.2 \mathrm{~Hz}), 7.08(2 \mathrm{H}, \mathrm{s})$; IR $\left(\mathrm{KBr}, v_{\max }\right.$, $\left.\mathrm{cm}^{-1}\right): 3501,3076,2970,2928,1606,1510,1465,1402,1320$, 1263, 1218, 1150, 1011, 934, 820, 792, 742, 576; HR-MS (ESI): Calcd. for $\mathrm{C}_{16} \mathrm{H}_{18} \mathrm{NO}_{2} \mathrm{Br}_{2}[\mathrm{M}+\mathrm{H}]^{+}$: 413.9705, 415.9685, 417.9664; Found: 413.9698, 415.9706, 417.9661.

2,6-Dibromo-4-[2-chloro-4-(diethylamino)phenoxy]phenol (7d): Yellow solid; yield: $54.3 \%$; m.p.: 106-108 ${ }^{\circ} \mathrm{C}$; ${ }^{1} \mathrm{H}$ NMR spectrum (400 MHz; $\mathrm{d}_{1}-\mathrm{CDCl}_{3}$; TMS): $\delta(\mathrm{ppm})=$ $1.18(6 \mathrm{H}, \mathrm{t}, J=7.2 \mathrm{~Hz}), 3.33(4 \mathrm{H}, \mathrm{q}, J=7.2 \mathrm{~Hz}), 5.57(1 \mathrm{H}, \mathrm{s})$, $6.53(1 \mathrm{H}, \mathrm{d}, J=8.8 \mathrm{~Hz}), 6.68(1 \mathrm{H}, \mathrm{d}, J=2.4 \mathrm{~Hz}), 6.91(1 \mathrm{H}, \mathrm{d}$, $J=8.8 \mathrm{~Hz}), 7.02(2 \mathrm{H}, \mathrm{s})$; IR $\left(\mathrm{KBr}, \mathrm{v}_{\max }, \mathrm{cm}^{-1}\right): 3517,3073$, 2969, 2927, 1607, 1562, 1503, 1466, 1403, 1356, 1318, 1276, 1233, 1196, 1150, 1071, 1042, 1016, 930, 800, 752, 695, 580, 436; HR-MS (ESI): Calcd. for $\mathrm{C}_{16} \mathrm{H}_{17} \mathrm{NO}_{2} \mathrm{Br}_{2} \mathrm{Cl}[\mathrm{M}+\mathrm{H}]^{+}$: 447.9315, 449.9295, 451.9274; Found: 447.9305, 449.9284, 451.9267 .

2,6-Dibromo-4-[4-(dipropylamino)phenoxy]phenol (7e): Yellow oilyliquid; yield: $48.7 \% ;{ }^{1} \mathrm{H}$ NMR spectrum $\left(400 \mathrm{MHz} ; \mathrm{d}_{1}-\mathrm{CDCl}_{3} ; \mathrm{TMS}\right): \delta(\mathrm{ppm})=0.93(6 \mathrm{H}, \mathrm{t}, J=7.2$ $\mathrm{Hz}), 1.63(4 \mathrm{H}, \mathrm{m}, J=7.6 \mathrm{~Hz}), 3.22(4 \mathrm{H}, \mathrm{t}, J=7.6 \mathrm{~Hz}), 5.55$ $(1 \mathrm{H}, \mathrm{s}), 6.60(2 \mathrm{H}, \mathrm{d}, J=9.2 \mathrm{~Hz}), 6.86(2 \mathrm{H}, \mathrm{d}, J=9.2 \mathrm{~Hz})$, 7.07 (2H, s); IR (KBr, $\left.v_{\max }, \mathrm{cm}^{-1}\right): 3505,3077,2960,2872$, 1607, 1565, 1510, 1465, 1402, 1321, 1218, 1150, 1101, 1047, 932, 819, 744, 549; HR-MS (ESI): Calcd. for $\mathrm{C}_{18} \mathrm{H}_{22} \mathrm{NO}_{2} \mathrm{Br}_{2}$ $[\mathrm{M}+\mathrm{H}]^{+}:$442.0018, 443.9998, 445.9977; Found: 442.0013, 443.9999, 445.9976.

2,6-Dibromo-4-[4-(dibutylamino)phenoxy]phenol (7f): Yellow oilyliquid; yield: $40.2 \%$; ${ }^{1} \mathrm{H}$ NMR spectrum $(400 \mathrm{MHz}$; $\mathrm{d}_{1}-\mathrm{CDCl}_{3}$; TMS): $\delta(\mathrm{ppm})=0.96(6 \mathrm{H}, \mathrm{t}, J=7.6 \mathrm{~Hz}), 1.36(4 \mathrm{H}$, $\mathrm{m}), 1.57(4 \mathrm{H}, \mathrm{m}), 3.24(4 \mathrm{H}, \mathrm{t}, J=7.6 \mathrm{~Hz}), 5.56(1 \mathrm{H}, \mathrm{s}), 6.60$ $(2 \mathrm{H}, \mathrm{d}, J=8.8 \mathrm{~Hz}), 6.86(2 \mathrm{H}, \mathrm{d}, J=8.8 \mathrm{~Hz}), 7.08(2 \mathrm{H}, \mathrm{s})$; IR $\left(\mathrm{KBr}, \mathrm{v}_{\max }, \mathrm{cm}^{-1}\right): 3511,3078,3044,2956,2866,1608,1565$, 1510, 1465, 1402, 1369, 1321, 1215, 1149, 1109, 1051, 1008, 931, 818, 782, 744, 697, 578; HR-MS (ESI): Calcd. for $\mathrm{C}_{20} \mathrm{H}_{26} \mathrm{NO}_{2} \mathrm{Br}_{2}[\mathrm{M}+\mathrm{H}]^{+}:$470.0331, 472.0311, 474.0290; Found: 470.0334, 472.0306, 474.0297.

2,6-Dibromo-4-[5-bromo-2-chloro-4-(dibenzylamino)phenoxy]phenol (7g): White solid; yield: $49.9 \%$, m.p.: 180$182{ }^{\circ} \mathrm{C} ;{ }^{1} \mathrm{H}$ NMR spectrum (400 MHz; $\left.\mathrm{d}_{1}-\mathrm{CDCl}_{3} ; \mathrm{TMS}\right): \delta$ $(\mathrm{ppm})=4.67(4 \mathrm{H}, \mathrm{s}), 5.64(1 \mathrm{H}, \mathrm{s}), 7.02(1 \mathrm{H}, \mathrm{s}), 7.08(3 \mathrm{H}, \mathrm{d}, J$ $=6.8 \mathrm{~Hz}), 7.15-7.29(10 \mathrm{H}, \mathrm{m})$; IR $\left(\mathrm{KBr}, \mathrm{v}_{\max }, \mathrm{cm}^{-1}\right): 3476$, 3381, 3074, 2923, 2853, 1605, 1568, 1451, 1409, 1383, 1309, 1278, 1218, 1189, 1155, 1067, 973, 922, 848, 779, 737, 713, 582, 532, 493, 454; HR-MS (ESI): Calcd. for $\mathrm{C}_{26} \mathrm{H}_{20} \mathrm{NO}_{2} \mathrm{Br}{ }_{2} \mathrm{Cl}$ 
$[\mathrm{M}+\mathrm{H}]^{+}:$649.8733, 651.8713, 653.8693; Found: 649.8718, 651.8699, 653.8683.

2,6-Dibromo-4-[2-bromo-4-(dimethylamino)phenoxy]phenol (7h): White solid; yield: $45.4 \%$, m.p.: $120-122{ }^{\circ} \mathrm{C} ;{ }^{1} \mathrm{H}$ NMR spectrum (400 MHz; $\left.\mathrm{d}_{1}-\mathrm{CDCl}_{3} ; \mathrm{TMS}\right): \delta(\mathrm{ppm})=2.78$ $(6 \mathrm{H}, \mathrm{s}), 5.70(1 \mathrm{H}, \mathrm{s}), 6.90\left(1 \mathrm{H}, \mathrm{dd}, J_{1}=8.8 \mathrm{~Hz}, J_{2}=2.8 \mathrm{~Hz}\right)$, $7.08(1 \mathrm{H}, \mathrm{d}, J=8.8 \mathrm{~Hz}), 7.14(1 \mathrm{H}, \mathrm{s}), 7.2(2 \mathrm{H}, \mathrm{d}, J=2.8 \mathrm{~Hz})$; IR $\left(\mathrm{KBr}, \mathrm{v}_{\max }, \mathrm{cm}^{-1}\right): 3418,3078,2954,1586,1559,1464$, 1403, 1338, 1263, 1213, 1153, 1034, 937, 864, 789, 752, 669,584, 544; HR-MS (ESI): Calcd. for $\mathrm{C}_{14} \mathrm{H}_{13} \mathrm{NO}_{2} \mathrm{Br}_{3}[\mathrm{M}+\mathrm{H}]^{+}$: 463.8497, 465.8477, 466.8456; Found: 463.8488, 465.8466, 466.8443 .

Biological assay: Wild Staphylcoccus aures SC and standard Staphylcoccus aures ATCC26112 were used in the studies. The antibacterial activities of the target compounds in vitro were tested via agar-well diffusion method. Every sample $(1000 \mu \mathrm{g})$ was dissolved with ethanol $(1 \mathrm{~mL})$ and diluted to $50 \mu \mathrm{g} / \mathrm{mL}$ with ethanol. The resulting solution was added to the flat plate of an Oxford cup which was covered with culture and kept at $37^{\circ} \mathrm{C}$ for $24 \mathrm{~h}$. The results of average diameters of the bacteriostatic circle are listed in Table- 1 .

\section{RESULTS AND DISCUSSION}

The bioactivities of compounds $\mathbf{7 a - h}$ against $S$. aureus ATCC26112 and S. aureus SC are shown in Table-1. The results showed that all target compounds exhibited antibacterial activity at the concentration of $50.0 \mu \mathrm{g} / \mathrm{mL}$.

\section{TABLE-1}

ANTIBACTERIAL ACTIVITY OF THE TARGET COMPOUNDS (7a-h) AT THE CONCENTRATION OF $50.0 \mu \mathrm{g} / \mathrm{mL}$ TOWARD S. aureus ATCC26112 and S. aureus SC

\begin{tabular}{|c|c|c|}
\hline \multirow{2}{*}{ Compound } & \multicolumn{2}{|c|}{ Diameter of inhibition zone (mm) } \\
\hline & S. aureus ATCC26112 & S. aureus $S C$ \\
\hline $7 \mathbf{a}$ & 11 & 13 \\
\hline $7 b$ & 9 & 10 \\
\hline $7 c$ & 8 & 8 \\
\hline 7d & 11.5 & 11 \\
\hline $7 e$ & 13 & 12 \\
\hline $7 f$ & 7 & 9 \\
\hline $7 \mathrm{~g}$ & 7 & 9 \\
\hline $7 \mathrm{~h}$ & 8.5 & 10 \\
\hline Triclosan & 22 & 20 \\
\hline Ethanol $^{\mathrm{b}}$ & - & - \\
\hline
\end{tabular}

During the preparation of $\mathbf{5 a - g}$, by-products increased as the reduction of activities of $\mathrm{RBr}$. So it's necessary to increase reaction temperature and time. Otherwise, $\mathrm{RBr}$ were vapourized along with long-playing heating, so more $\mathrm{RBr}$ needed during reaction process.

As shown in Scheme-I, the intermediates 6a-g were synthesized by $\mathbf{5 a - g}$ with $\mathrm{HBr}$ in HAc. On this condition the benzyl located in the nitrogen would fall off partially, so the yield of the intermediate $\mathbf{6 g}$ was very low.

\section{Conclusion}

In conclusion, eight novel brominated hydroxy diphenyl ethers derivatives were designed and synthesized. Compared to 4-hydroxy (Fig. 1, 5) diphenyl ethers derivatives ${ }^{15}$ with nitro group which exhibited good antibacterial activity at the concentration of $10 \mu \mathrm{g} / \mathrm{mL}$, all target compounds have similar structures with them, but their antibacterial activities are much worse. This made a further proof that nitro group is important for enhancing antibacterial activitiy. We will continue to study structure-activity relations of brominated hydroxy diphenyl ethers derivatives.

\section{ACKNOWLEDGEMENTS}

The authors appreciated the financial support from the National Science Foundation of China (No. 21072135), the antibacterial activity test of Chengdu Medical College (cx20100037) and the ${ }^{1} \mathrm{H}$ NMR analysis by Sichuan University Analytical \& Testing Center.

\section{REFERENCES}

1. G.M. Sharma, B. Vig and P.R. Burkholder, Marine Technology Society, Washington D.C. (1969).

2. G.M. Sharma and B. Vig, Tetrahedron Lett., 13, 1715 (1972).

3. B. Carté and D.J. Faulkner, Tetrahedron, 37, 2335 (1981).

4. R.S. Norton, K.D. Croft and R.J. Wells, Tetrahedron, 37, 2341 (1981).

5. E.N. Segraves, R.R. Shah, N.L. Segraves, T.A. Johnson, S. Whitman, J.K. Sui, V.A. Kenyon, R.H. Cichewicz, P. Crews and T.R. Holman, J. Med. Chem., 47, 4060 (2004).

6. J. Salvá and D.J. Faulkner, J. Nat. Prod., 53, 757 (1990).

7. D. Handayani, R.A. Edrada, P. Proksch, V. Wray, L. Witte, R.W.M. Van Soest and A. Kunzmann, J. Nat. Prod., 60, 1313 (1997).

8. Y.L. Wang, M.L. Ma and S.H. Chen, Youji Huaxue, 25, 734 (2005).

9. K.Q. Wu, Y.L. Wang and H. Zheng, CN 1597657 (2005).

10. H. Jin, S. Chen and R.T. Hou, Youji Huaxue, 26, 1424 (2006).

11. S. Chen, Y. Xin and Y. Zhan, Youji Huaxue, 3, 498 (2008).

12. N.K. Utkina, V,A. Denisenko, O.V. Scholokova, M.V. Virovaya, A.V. Gerasimenko, D.Y. Popov, V.B. Krasokhin and A.M. Popov, J. Nat. Prod., 64, 151 (2001)

13. N.K. Utkina, V.A. Denisenko, M,V. Virovaya, O,V. Scholokova and N.G. Prokof'eva, J. Nat. Prod., 65, 1213 (2002).

14. X. Tang, M. Xie, Y.X. Sun, J.H. Liu, Z.C. Zhong and Y.L. Wang, Chin. Chem. Lett., 20, 435 (2009).

15. Y. Zou, J. Wu and R.T. Hou, Youji Huaxue, 1, 111( 2008).

16. Z.X. Yang, H. Chen and Y.L. Wang, Youji Huaxue, 3, 432 (2008).

17. T. Saitoh and J. Ichikawa, J. Am. Chem. Soc., 127, 9696 (2005). 